\title{
THE EXISTENCE OF OSCILLATORY SOLUTIONS FOR THE EQUATION
}

\author{
$d^{2} y / d t^{2}+q(t) y^{r}=0,0<r<1$ \\ KUO-LIANG CHIOU
}

\begin{abstract}
This paper gives sufficient conditions for the existence of oscillatory solutions in the sublinear case of the second order differential equation $d^{2} y \mid d t^{2}+q(t) y^{r}=0$, where $q(t)$ is nonnegative and continuous and $0<r<1$. We use the technique of [3, Theorem 3.1] and obtain a result which extends [2, Corollary 1], [3, Theorem 3.1], and [3, Theorem 3.2].
\end{abstract}

We are here concerned with the oscillatory behavior of solutions of the following second order nonlinear differential equation:

$$
d^{2} y / d t^{2}+q(t) y^{r}=0
$$

where $q(t) \geqq 0$ and continuous on $(0, \infty)$ and $\Gamma$ satisfies $0<r=p / q<1$ where $p, q$ are odd integers.

It will be tacitly assumed here that every locally defined solution of (1) is continuously extendable throughout the entire nonnegative real axis. A nontrivial solution $y(t)$ of $(1)$ is said to be oscillatory if for any positive number $a$ there exists $b$ greater than $a$ such that $y(b)=0$.

For the sake of completeness we state some related results. Belohorec [1] has shown the following result on the existence of one oscillatory solution.

THEOREM A. If $(d / d t)\left(q(t) t^{(r+3) / 2}\right) \leqq 0$ and $q(t) t^{(r+3) / 2} \geqq K_{1}>0$ for $t>0$, then (1) has oscillatory solutions.

Coffman and Wong [2] obtained a result which is an improvement of the result of Theorem A. namely,

THEOREM B. If $q(t) t^{(r+3) / 2}(\log t)^{u}$ is nonincreasing for some $u \leqq 0$ and bounded away from 0 , then equation (1) has an oscillatory solution.

Very recently, Heidel and Hinton [3] have other results for equation (1), namely,

Received by the editors November 29, 1971 and, in revised form, December 20, 1971. AMS 1970 subject classifications. Primary 34C10.

Key words and phrases. Oscillation. 
THEOREM C. If $(d / d t)\left(q(t) t^{(r+3) / 2}\right) \geqq 0$ and $q(t) t^{(r+3) / 2} \leqq K$ for $t>0$, then every solution $y(t)$ of (1) such that $y\left(t_{0}\right)=0, t_{0}>0$, and $\left|y^{\prime}\left(t_{0}\right)\right|$ is sufficiently small is oscillatory.

THEOREM D. If $\lim _{t \rightarrow \infty} t(d / d t)\left(q(t) t^{(r+3) / 2}\right)=\infty$ and $(d / d t)\left(q(t) t^{(r+3) / 2}\right) \geqq 0$ for $t>0$, then every solution $y(t)$ of.(1) such that $y\left(t_{0}\right)=0, t_{0}>0$, and $\left|y^{\prime}\left(t_{0}\right)\right|$ is sufficiently small is oscillatory.

The purpose of this paper is to show that Theorem C and Theorem D remain valid without the assumption $q(t) t^{(r+3) / 2} \leqq K$ for $t>0$ in Theorem C, and the assumption $\lim _{t \rightarrow \infty} t(d / d t)\left(q(t) t^{(r+3) / 2}\right)=\infty$ in Theorem $\mathrm{D}$. This will unify Theorems $\mathrm{C}$ and $\mathrm{D}$ into a single criterion for the existence of oscillatory solutions.

We can now state our theorems which will be proved by refining the technique of [3].

THEOREM 1. If $(d / d t)\left(q(t) t^{(r+3) / 2}\right) \geqq 0$ for $t>0$, then every solution $y(t)$ of (1) such that $y\left(t_{0}\right)=0, t_{0}>0$, and $\left|y^{\prime}\left(t_{0}\right)\right|$ is sufficiently small is oscillatory.

Proof. We make the change of variables, $x=\log t, y(t)=t^{1 / 2} w(x)$, which transforms (1) into

$$
w^{\prime \prime}-\frac{1}{4} w+f(x) w^{r}=0, \quad{ }^{\prime}=d / d x,
$$

where $f(x)=q(t) t^{(r+3) / 2}$. Clearly, the $(0, \infty) t$-interval corresponds to the $(-\infty, \infty) x$-interval. Define $\beta(x)=(4 f(x))^{1 /(1-r)}$ and $G(w(x))$ by

$$
G(w(x))=w^{\prime 2}(x) / 2+\left(f(x) w(x)^{r+1}\right) /(r+1)-w^{2}(x) / 8 .
$$

Then $G(w(x))=G\left(w\left(x_{0}\right)\right)+\left(\int_{x_{0}}^{x} f^{\prime}(u) w(u)^{r+1} d u\right) /(r+1)$.

We claim that if $w\left(x_{0}\right)=0$ and $w^{\prime 2}\left(x_{0}\right) / 2<K f\left(x_{0}\right)^{2 /(1-r)}, \quad K=c^{1+r}$ $\cdot 4^{(1+r) /(1-r)} \cdot(1-r) / 2(1+r), c$ is a constant and $0<c<1$, then $|w(x)|<c \beta(x)$ for $x \geqq x_{0}$. As long as $|w(x)| \leqq c \beta(x)$, then $w^{r+1}<c^{r+1} \cdot(4 f(x))^{(1+r) /(1-r)}$. Hence

$$
G(w(x)) \leqq w^{\prime 2}\left(x_{0}\right) / 2-K f\left(x_{0}\right)^{2 /(1-r)}+K f(x)^{2 /(1-r)}<K f(x)^{2 /(1-r)}
$$

Suppose that $|w(x)|=c \beta(x)$ for some $x>x_{0}$ and let $x_{1}>x_{0}$ be the first such point. Then

$$
\begin{aligned}
G\left(w\left(x_{1}\right)\right)= & w^{\prime 2}\left(x_{1}\right) / 2+\left(f\left(x_{1}\right) c^{r+1}\left(4 f\left(x_{1}\right)\right)^{(1+r) /(1-r)}\right) /(r+1) \\
& -\left(c^{2}\left(4 f\left(x_{1}\right)\right)^{2 /(1-r)}\right) / 8 \\
\geqq & w^{\prime 2}\left(x_{1}\right) / 2+K f\left(x_{1}\right)^{2 /(1-r)} \geqq K f\left(x_{1}\right)^{2 /(1-r)},
\end{aligned}
$$

since $0<c<1,0<r<1$, and $w^{\prime 2}\left(x_{1}\right) / 2>0$. But this contradicts $G(w(x))<$ $K f(x)^{2 /(1-r)}$ for $x_{0} \leqq x \leqq x_{1}$. Therefore, $|w(x)|<c \beta(x)$ for $x \geqq x_{0}$.

Transforming back to $t$ variables we obtain

$$
\left(y(t) / t^{1 / 2}\right)^{1-r}<c^{1-r}\left(4 q(t) t^{(r \rightarrow 3) / 2}\right) \quad \text { for large } t .
$$


Therefore, $q(t) y(t)^{r-1}>c^{r-1} /\left(4 t^{2}\right)=(1+\varepsilon) /\left(4 t^{2}\right)$ for some $\varepsilon>0$ and for large $t$. Thus $y(t)$ must be an oscillatory solution of $d^{2} y / d t^{2}+\left(q(t) y(t)^{r-1}\right) y=$ 0 . Therefore, the theorem is proved.

In the proof of the above theorem we use $(d / d t)\left(q(t) t^{(r+3) / 2}\right) \geqq 0$ to show that $G(w(x))<K f(x)^{2 /(1-r)}$ for $|w(x)| \leqq c \beta(x)$. We can also assert that this statement is valid if $(d / d t)\left(q(t) t^{(r+3) / 2}\right) \leqq 0$ and $q(t) t^{(r+3) / 2} \geqq K_{1}>0$ for $t>0$. Therefore, we have the following alternate proof of Belohorec's result, Theorem A.

THEOREM 2. If $(d / d t)\left(q(t) t^{(r+3) / 2}\right) \leqq 0$ and $q(t) t^{(r+3) / 2} \geqq K_{1}>0$ for $t>0$, then every solution $y(t)$ of (1) such that $t\left(y_{0}\right)=0, t_{0}>0$, and $\left|y^{\prime}\left(t_{0}\right)\right|$ is sufficiently small is oscillatory.

Proof. The proof of this theorem will follow the proof of Theorem 1. In this theorem we may choose $w^{\prime 2}\left(x_{0}\right) / 2<K K_{1}^{2 /(1-r)}$ where $K$ is the same as before and $0<K_{1} \leqq q(t) t^{(r+3) / 2}, t>0$.

We assert that $G(w(x))<K f(x)^{2 /(1-r)}$ as long as $|w(x)| \leqq c \beta(x)$. To see this, consider

$$
\begin{aligned}
G(w(x)) & =G\left(w\left(x_{0}\right)\right)+\int_{x_{0}}^{x}\left(w(u)^{r+1} f !(u) /(r+1)\right) d u \\
& \leqq w^{\prime 2}\left(x_{0}\right) / 2<K K_{1}^{2 /(1-r)} \leqq K f(x)^{2 /(1-r)}
\end{aligned}
$$

since $f^{\prime}(u) \leqq 0$ and $K_{1} \leqq f(x)$. This proves the assertion.

Then, we may follow the proof of Theorem 1 to obtain the desired result.

REMARK. In Theorem 1 if we replace $(d / d t)\left(q(t) t^{(r+3) / 2}\right) \geqq 0$ by the nondecreasing function $q(t) t^{(r+3) / 2}$, then Theorem 1 is still valid. Similarly, in Theorem 2 if we interchange $(d / d t)\left(q(t) t^{(r+3) / 2}\right) \leqq 0$ and the nonincreasing function $q(t) t^{(r+3) / 2}$, then Theorem 2 is also valid.

ACKNOWLEDGEMENT. The author would like to thank Dr. J. W. Heidel for his valuable suggestions and comments. The author would also like to thank the referee for his suggestion of this paper.

\section{REFERENCES}

1. S. Belohorec, On some properties of the equation $y^{\prime \prime}(x)+f(x) y^{x}(x)=0,0<\alpha<1$, Mat. Casopis Sloven. Akad. Vied. 17 (1967), 10-19. MR 35 \#5703.

2. C. V. Coffman and J. S. W. Wong, Second order nonlinear oscillations, Bull. Amer. Math. Soc. 75 (1969), 1379-1382. MR 40 \#449.

3. J. W. Heidel and Don B. Hinton, The existence of oscillatory solutions for a nonlinear differential equation, SIAM J. Math. Anal. (to appear).

Department of Mathematics, University of Tennessee, Knoxville, Tennessee 37916 